\title{
Stochastic Unit Commitment Problem with Security and Emissions Constraints
}

\author{
Rui Laia ${ }^{1,2}$, Hugo M.I. Pousinho ${ }^{1,2}$, Rui Melício ${ }^{1,2}$, Victor M.F. Mendes ${ }^{1,3}$, \\ and Manuel Collares-Pereira ${ }^{1}$ \\ ${ }^{1}$ University of Évora, Évora, Portugal \\ \{rui.j.laia, hpousinho\}@gmail.com, \\ \{ruimelicio, collarespereira\}@uevora.pt \\ ${ }^{2}$ IDMEC/LAETA, Instituto Superior Técnico, Universidade de Lisboa, Lisbon, Portugal \\ ${ }^{3}$ Instituto Superior of Engenharia de Lisboa, Lisbon, Portugal \\ vfmendes@deea.isel.pt
}

\begin{abstract}
This paper presents a stochastic optimization-based approach for the unit commitment (UC) problem under uncertainty on a deregulated electricity market that includes day-ahead bidding and bilateral contracts. The market uncertainty is modeled via price scenarios so as to find the optimal schedule. An efficient mixed-integer linear program is proposed for the UC problem, considering not only operational constraints including security ones on units, but also emission allowance constraints. Emission allowances are used to mitigate carbon footprint during the operation of units. While security constraints settle on spinning reserve are used to provide reliable bidding strategies. Numerical results from a case study are presented to show the effectiveness of the approach.
\end{abstract}

Keywords: Emission allowances, stochastic optimization, security constraints, unit commitment.

\section{Introduction}

The UC is one of the most challenging optimization problems in power system operation, which has deserved an increasing interest due to today's energy shortage [1]. In a deregulated electricity market, the generation companies (GENCOs) operate under a high competition degree due to the nodal variations of electricity prices [2] in order to obtain the best profit bidding in the day-ahead market and bilateral contracting. So, the optimal schedule of the thermal units must consider the electricity prices uncertainty and other requirements, such as: technical operating constraints including security ones on the units and environmental constraints required to ensure admissible emission allowance levels. Multiple deterministic approaches have been proposed to solve the UC problem, which can be categorized into: priorities list, classical mathematical programming methods and intelligent methods [3]. Within the classical mathematical programming methods, mixed-integer linear programming (MILP) has been broadly applied for solving the UC problem [4], due to the suitable 
proprieties to guarantee global optimality and support the management decision. However, the literature about UC problem tends to address the problem without security and emission constraints uncertainty [5]. Security constraints provide reliable bidding strategies, for instance, spinning reserve levels recommended by the Union for the Coordination of the Transmission of Electricity [6]. Hence, this paper proposes a stochastic MILP approach to handle the electricity price uncertainty and solve the UC problem considering appropriate constraints to address a more realistic and feasible results for the management of thermal units in a competitive electricity market environment. A case study is presented for a schedule over a time horizon of 24 hours with hourly periods.

\section{Relationship to Collective Awareness Systems}

The technological evolution on electric power system encouraged by the expansion of distributed generation has been crucial to create collective awareness systems useful to define new energy consumption and production patterns. A collective awareness system can result from the development of powerful optimization approaches for the management of energy systems, helping to make decisions. The collective awareness system not only promotes the sustainable use of energy resources in favor of an effective low-carbon economy [7], but also processes the optimal decision. In order to achieve this optimal decision, collective tools are essential to provide real-time information on market data such as, the electricity prices, bilateral contracts and admissible emission allowance levels, allowing a GENCO to maximize the expected profit. Hence, research on technological innovation for collective tools based on approaches for solving the UC problem of a GENCO is crucial to achieve guidelines for the best bidding in an electricity market.

\section{State of the Art}

A review of literature describing the UC problem of a GENCO reveals that this problem has been treated in some way by avowing stochastic modeling, i.e., ignoring the random event on the electricity market [5]. Such treatment cannot provide a convenient level of precautions on the decision. This treatment is not appropriated, because in nowadays the most certainty thing for a GENCO is the uncertainty. Optimization methods for solving the UC problem have been addressed since the old priorities list method [8] to the classical mathematical programming methods until the more recently reported artificial intelligence methods [9]. Although, easy to implement and requiring a small computation time, the priority list method does not ensures an economic convenient solution near a global optimal one, implying a higher operation cost [8]. Within the classical methods are included dynamic programming (DP), linear programming, nonlinear programming and Lagrangian relaxation-based techniques [10]. DP methods are flexible but suffer from the "curse of dimensionality", due to the increase in the problem size related with the number of thermal units to be committed and the number of states considered for modeling the 
thermal behavior of each unit, implying an eventually huge use of computation memory and processing time. Although the Lagrangian relaxation [11] can overcome the previous limitation, does not always lead to a conveniently feasible solution, requiring in order to set a feasible solution the satisfaction of some violated constraints using heuristics, undermining the optimality. Artificial intelligence (AI) methods based on artificial neural networks [12], genetic algorithms [13], and evolutionary algorithms [9] have also been applied. However, the major limitation of the AI methods is the likelihood to obtain a convenient solution near global optimum, especially with a few thermal units. MILP has been applied with success for solving the UC problem [14]. Although, nonlinear constraints have to be converted into linear ones by piecewise linear approximation, MILP allows an easily inclusion of new constraints that makes the formulation of the problem more appropriated in order to conveniently support the management decision.

The literature about the UC problem of a GENCO tends to address the problem without security and emission constraints uncertainty. The emission constraints that can significantly affect the solutions of UC problem cannot be disregarded in the present context of the regulation. For instance, the authors in [5] address the problem of modeling the emission constraints into the UC, but with no uncertainty modeled. Hence, this paper as a contribution proposes a stochastic MILP approach to handle the electricity price uncertainty and solve the UC problem considering appropriate constraints, addressing a more realistic and feasible results for the management of thermal units in a competitive electricity market environment.

\section{Problem Formulation}

The UC problem can be stated as to find the schedule on status and the power generated for each thermal unit $i$ at each time period $t$ that optimizes performance criterion, involving market trading revenue and costs subject to a set of constraints on security, emissions and operation of the units.

\subsection{Objective Function}

The UC problem of a price-taker GENCO on price uncertainty has an objective function given by a measure of the expected profit attained by the sales of energy in a day-ahead market with bilateral contracts. The objective function to be maximized can be stated as:

$$
\sum_{t=1}^{T} \sum_{m=1}^{M} \lambda_{m t}^{b c} p_{m t}^{b c}+\sum_{\omega=1}^{\Omega} \rho_{\omega}\left\{\sum_{t=1}^{T} \lambda_{\omega t}^{b} p_{\omega t}^{b}-\sum_{i=1}^{I} F_{\omega i t}\right\}
$$

The objective function in (1) is composed of two terms, namely: the revenue from selling through bilateral contracts between the GENCO and other market entities; the expected profit obtained by GENCO from selling its production in the day-ahead market minus the incurred operating costs. In (1), $\lambda_{m t}^{b c}$ is the electricity price at 
period $t$ for the bilateral contract $m ; p_{m t}^{b c}$ is the power at period $t$ for the bilateral contract $m ; \rho_{\omega}$ is the probability of occurrence for the scenario $\omega ; \lambda_{\omega t}^{b}$ is the electricity price at period $t$ for the scenario $\omega$; and $p_{\omega t}^{b}$ is the power to bid in the dayahead market at period $t$ for the scenario $\omega$. At each period, the operating costs, $F_{\omega i t}$ can be stated as:

$$
F_{\omega i t}=A_{i} u_{\omega i t}+d_{\omega i t}+b_{\omega i t}+C_{i} z_{\omega i t} \quad \forall \omega, \quad \forall i, \quad \forall t
$$

The operating costs in (2) are composed of four terms, namely: the fixed cost, $A_{i}$, variable cost, $d_{\omega i t}$, start-up cost, $b_{\omega i t}$, and shut-down cost, $C_{i}$, of the units.

\subsection{Constraints}

The optimization problem is subject to a set of constraints due to the modeling. The modeling for the variable cost function by piecewise linear approximation introduces the constraints stated as:

$$
\begin{aligned}
& d_{\omega i t}=\sum_{l=1}^{L} F_{i}^{l} \delta_{\omega i t}^{l} \quad \forall \omega, \quad \forall i, \quad \forall t \\
& p_{\omega i t}=p_{i}^{\min } u_{\omega i t}+\sum_{l=1}^{L} \delta_{\omega i t}^{l} \quad \forall \omega, \quad \forall i, \quad \forall t \\
& \left(T_{i}^{1}-p_{i}^{\min }\right) t_{\omega i t}^{1} \leq \delta_{\omega i t}^{1} \quad \forall \omega, \quad \forall i, \quad \forall t \\
& \delta_{\omega i t}^{1} \leq\left(T_{i}^{1}-p_{i}^{\min }\right) u_{\omega i t} \quad \forall \omega, \quad \forall i, \quad \forall t \\
& \left(T_{i}^{l}-T_{i}^{l-1}\right) t_{\omega i t}^{l} \leq \delta_{\omega i t}^{l} \quad \forall \omega, \quad \forall i, \quad \forall t, \quad \forall l=2, \ldots, L-1 \\
& \delta_{\omega i t}^{l} \leq\left(T_{i}^{l}-T_{i}^{l-1}\right) t_{\omega i t}^{l-1} \quad \forall \omega, \quad \forall i, \quad \forall t, \quad \forall l=2, \ldots, L-1 \\
& 0 \leq \delta_{\omega i t}^{L} \leq\left(p_{i}^{\max }-T_{\omega i t}^{L-1}\right) t_{\omega i t}^{L-1} \quad \forall \omega, \quad \forall i, \quad \forall t
\end{aligned}
$$

In (4), the power generation of the unit $i$ is given by the minimum power generation plus the sum of the power $\delta_{\omega i t}^{l}$ associated with each segment $l$. The binary variable $u_{\omega i t}$ ensures that the power generation is equal to 0 if the unit $i$ is offline. In (5)-(9), the limits of the power generated in each segment are set. This power must be between zero and the maximum size of each segment. This is assured with a binary variable, $t_{\omega i t}^{l}$, which is equal to 1 if the power generation of the unit at period $t$ has exceeded segment $l$.

The modeling for the start-up cost is given by a stairwise linear approximation [15]. This linear approximation introduces the constraints stated as: 


$$
\begin{gathered}
b_{\omega i t} \geq K_{i}^{\beta}\left(u_{\omega i t}-\sum_{r=1}^{\beta} u_{\omega i t-r}\right) \quad \forall \omega, \quad \forall i, \quad \forall t \\
b_{\omega i t} \geq 0 \quad \forall \omega, \quad \forall i, \quad \forall t
\end{gathered}
$$

In (10), the expression in parentheses is equal to 1 if the unit $i$ is online at period $t$ and has been offline $\beta$ preceding hours.

The modeling to limit the power of unit $i$ introduces the constraints stated as:

$$
\begin{gathered}
p_{i}^{\min } u_{\omega i t} \leq p_{\omega i t} \leq p_{\omega i t}^{\max } \forall \omega, \quad \forall i, \quad \forall t \\
p_{\omega i t}^{\max } \leq p_{i}^{\max }\left(u_{\omega i t}-z_{\omega i t+1}\right)+S D_{i} z_{\omega i t+1} \quad \forall \omega, \quad \forall i, \quad \forall t \\
p_{\omega i t}^{\max } \leq p_{\omega i t-1}^{\max }+R U_{i} u_{\omega i t-1}+S U_{i} y_{\omega i t} \quad \forall \omega, \quad \forall i, \quad \forall t \\
p_{\omega i t-1}-p_{\omega i t} \leq R D_{i} u_{\omega i t}+S D_{i} z_{\omega i t} \quad \forall \omega, \quad \forall i, \quad \forall t
\end{gathered}
$$

In (12), the generating limits of the units are set. In (13)-(15), the relation between the start-up and shut-down variables of the unit are given, using binary variables and their weights. In (13) and (14), the upper bound of $p_{\text {wit }}^{\max }$ is set, which is the maximum available power of the unit. These constraints involve: unit's actual capacity, start-up, $S U_{i}$, and shut-down, $S D_{i}$, ramp rate limits, and ramp-up, $R U_{i}$, limit. In (15), the ramp-down, $R D_{i}$, and shut-down ramp rate limits are considered.

The modeling for the minimum down time in a linear formulation introduces the constraints stated as:

$$
\begin{gathered}
\sum_{i=1}^{J_{i}} u_{\omega i t}=0 \quad \forall \omega, \quad \forall i \\
\sum_{t=k}^{k+D T_{i}-1}\left(1-u_{\omega i t}\right) \geq D T_{i} z_{\omega i t} \quad \forall \omega, \quad \forall i, \quad \forall k=J_{i}+1 \ldots T-D T_{i}+1 \\
\sum_{t=k}^{T}\left(1-u_{\omega i t}-z_{\omega i t}\right) \geq 0 \quad \forall \omega, \quad \forall i, \quad \forall k=T-D T_{i}+2 \ldots T \\
J_{i}=\min \left\{T,\left(D T_{i}-s_{\omega i 0}\right)\left(1-u_{\omega i 0}\right)\right\}
\end{gathered}
$$

In (16)-(18), the minimum down time $D T_{i}$ imposes that unit $i$ have to be down by at least the minimum down time before startup.

The modeling for the minimum up time is introduced by linear constraints stated as:

$$
\sum_{i=1}^{N_{i}}\left(1-u_{\omega i t}\right)=0 \quad \forall \omega, \quad \forall i
$$




$$
\begin{gathered}
\sum_{i=k}^{k+U T_{i}-1} u_{\omega i t} \geq U T_{i} y_{\omega i t} \quad \forall \omega, \quad \forall i, \quad \forall k=N_{i}+1 \ldots T-U T_{i}+1 \\
\sum_{t=k}^{T}\left(u_{\omega i t}-z_{\omega i t}\right) \geq 0 \quad \forall \omega, \quad \forall i, \quad \forall k=T-U T_{i}+2 \ldots T \\
N_{i}=\min \left\{T,\left(U T_{i}-U_{\omega i 0}\right) u_{\omega i 0}\right\}
\end{gathered}
$$

In (19)-(21), the minimum up time $U T_{i}$ imposes that unit $i$ have to be on by at least the minimum up time before shutdown.

The relation between the binary variables to identify start-up, shutdown and prohibited operating zones is stated as:

$$
\begin{gathered}
y_{\omega i t}-z_{\omega i t}=u_{\omega i t}-u_{\omega i t-1} \quad \forall \omega, \quad \forall i, \quad \forall t \\
y_{\omega i t}+z_{\omega i t} \leq 1 \quad \forall \omega, \quad \forall i, \quad \forall t
\end{gathered}
$$

The modeling for the emission function, $E_{\omega i t}$, used to quantify the emission of a thermal unit, introduces the constraint stated as:

$$
E_{\omega i t}=A e_{i} u_{\omega i t}+\sum_{r=1}^{R} F e_{i}^{r} \delta e_{\omega i t}^{r} \quad \forall \omega, \quad \forall i, \quad \forall t
$$

The modeling for the emission allowance over the time horizon introduces the constraint stated as:

$$
\sum_{i=1}^{I} \sum_{t=1}^{T} E_{\omega i t} \leq E M S \quad \forall \omega
$$

In (25), the sum of the emissions associated with the committed units during the time horizon is set to a value not greater than the total emission allowance, EMS.

In order to ensure that GENCO is capable to accomplish their agreements, the balance of trade constraint can be stated as:

$$
\sum_{i=1}^{I} p_{\omega i t}=p_{\omega t}^{b}+\sum_{m=1}^{M} p_{m t}^{b c} \quad \forall \omega, \quad \forall t
$$

In (26), the total power generated by the units is given by the total contracted power plus the day-ahead market power in each period.

The system security is included considering the European network codes [6], which recommend a spinning reserve level available at all times to cover eventual contingencies.

Hence, the spinning reserve, $S R_{\omega t}$, necessary to ensure reliability, is stated as:

$$
S R_{\omega t}+\sum_{i=1}^{I} p_{\omega i t} \leq \sum_{i=1}^{I} p_{\omega i t}^{\max } \quad \forall \omega, \quad \forall t
$$




\section{$5 \quad$ Case Study}

The proposed stochastic MILP approach has been tested on a representative case study based on a GENCO with ten thermal units. The time horizon considered is one day, divided into 24 hourly intervals. The approach Wavelet-Neuro-Fuzzy hybrid [16] is elected to generate 30 price scenarios, using historical data from the Iberian electricity market. The GENCO has to satisfy a bilateral contract with power and prices at each period. The price scenarios and the bilateral contract are shown in Fig. 1.
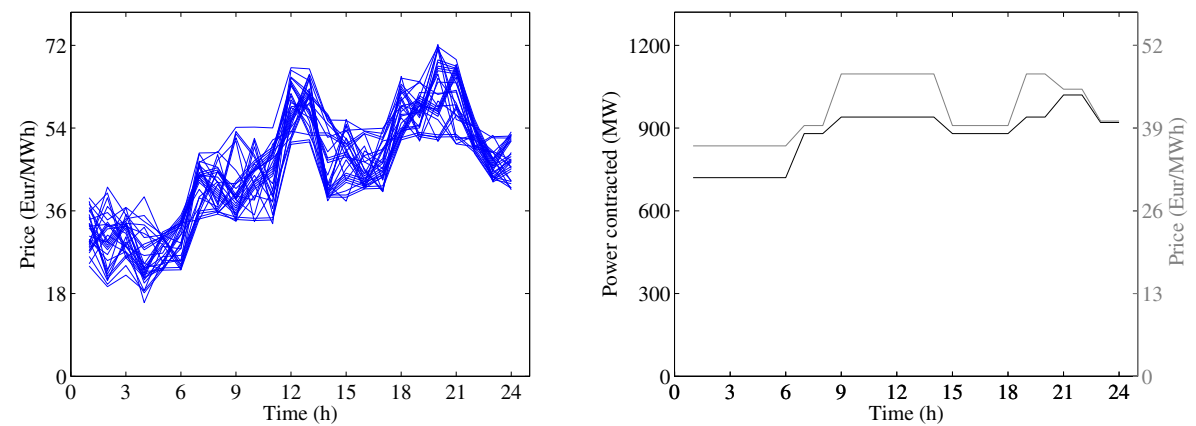

Fig. 1. Price scenarios (left) and power contracted and prices for bilateral contract (right)

The ten thermal units data, the minimum and maximum power, ramp up/down values, start-up and shut-down ramp rate values, minimum up/down time, fixed and shut-down costs are shown in Table 1.

Table 1. Thermal units' data

\begin{tabular}{ccccccccccc}
\hline Unit & $\begin{array}{c}p_{i}^{\min } \\
(\mathrm{MW})\end{array}$ & $\begin{array}{c}p_{i}^{\max } \\
(\mathrm{MW})\end{array}$ & $\begin{array}{c}R U_{i} \\
(\mathrm{MW})\end{array}$ & $\begin{array}{c}R D_{i} \\
(\mathrm{MW})\end{array}$ & $\begin{array}{c}S U_{i} \\
(\mathrm{MW})\end{array}$ & $\begin{array}{c}S D_{i} \\
(\mathrm{MW})\end{array}$ & $\begin{array}{c}U T_{i} \\
(\mathrm{~h})\end{array}$ & $\begin{array}{c}D T_{i} \\
(\mathrm{~h})\end{array}$ & $\begin{array}{c}A_{i} \\
(\text { Eur/h })\end{array}$ & $\begin{array}{c}C_{i} \\
(\text { Eur/h })\end{array}$ \\
\hline U1 & 45 & 85 & 35 & 35 & 60 & 70 & 8 & 3 & 2450 & 100 \\
$\mathrm{U} 2$ & 70 & 125 & 45 & 40 & 100 & 95 & 5 & 4 & 2900 & 170 \\
U3 & 110 & 160 & 60 & 50 & 125 & 140 & 8 & 4 & 3150 & 215 \\
U4 & 60 & 125 & 55 & 55 & 90 & 80 & 5 & 3 & 3060 & 120 \\
U5 & 90 & 170 & 40 & 60 & 100 & 100 & 6 & 3 & 2995 & 155 \\
U6 & 90 & 170 & 40 & 60 & 100 & 100 & 6 & 3 & 2995 & 155 \\
U7 & 80 & 145 & 35 & 40 & 90 & 105 & 9 & 6 & 3225 & 120 \\
U8 & 145 & 215 & 45 & 70 & 160 & 170 & 6 & 4 & 3810 & 110 \\
U9 & 200 & 380 & 60 & 50 & 230 & 250 & 10 & 6 & 4235 & 160 \\
U10 & 220 & 330 & 70 & 60 & 230 & 245 & 10 & 6 & 4490 & 135 \\
\hline
\end{tabular}


The variable costs of the thermal units have been modeled through piecewise linear approximations with three segments. The start-up costs are modeled through stairwise approximations with ten intervals. The thermal unit data associated with both models are available at [17].

The optimal hourly power generation for EMS $=200$ tons and EMS $=300$ tons for scenario 23 (\#23) is shown in Fig. 2.

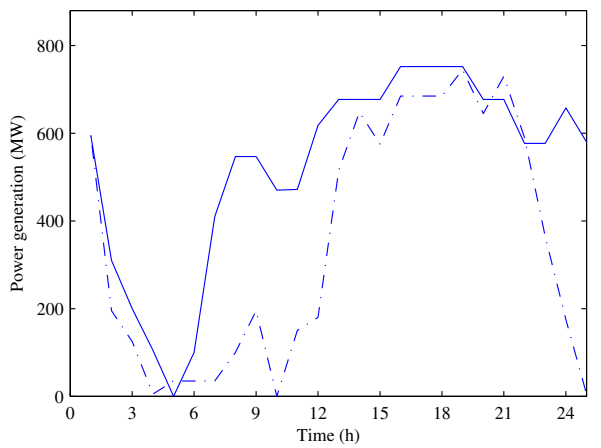

Fig. 2. Bidding power \#23: EMS = 200 tons (dashed-dotted line), EMS = 300 tons (solid line)

In Fig. 2, a comparison between EMS = 200 tons and EMS $=300$ tons, as expected, shows that the thermal system tends to operate at a high production level when a high emission allowance level is available. This operation is a compromise between the economic favoring and the level of the emission allowance in order to optimize the decision: when the emission allowance level is at a high value the production tends to follow the hourly price; otherwise, the production tends to be allocated in the hour with higher prices.

The hourly UC for the units at profiles EMS $=200$ tons and EMS $=300$ tons for \#23 are shown in Fig. 3.
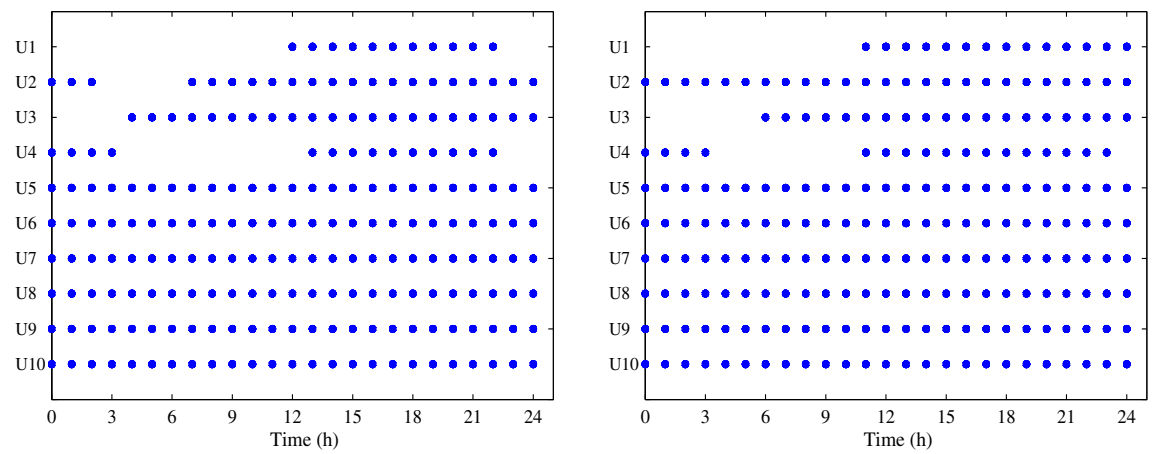

Fig. 3. Schedules \#23: EMS = 200 tons (left), EMS = 300 tons (right) 
In Fig. 3 is shown how the UC problem based on maximization of the expected profit is affected by the emission constraints. The UC of units U1, U2, U3 and U4 are affected by the different emission allowance levels. The number of units committed at each hour increases as the emission allowance level increases, as was expected.

The expected profit with and without spinning reserve for different emission allowance levels are shown in Table 2.

Table 2. Expected profit with and without spinning reserve and CPU time

\begin{tabular}{lccc}
\hline & Exp. profit with reserve (Eur) & Exp. profit without reserve (Eur) & CPU time (min) \\
\hline EMS $=200$ & 485,988 & 487,086 & 5.53 \\
$\mathrm{EMS}=250$ & 524,355 & 531,325 & 6.22 \\
$\mathrm{EMS}=300$ & 528,168 & 536,310 & 3.32 \\
\hline
\end{tabular}

In Table 2, a comparison of the expected profit with and without spinning reserve shows that the profit is higher when the constraint for the spinning reserve is not considered, as expected. The difference between the two profits is the cost of the spinning reserve. This difference is a contribution to decision-making in order to value this reserve.

\section{Conclusions}

A stochastic MILP approach for solving the UC problem of a price-taker thermal and emission constrained GENCO is presented, giving as main results: the short-term bidding strategies and the optimal schedule of the thermal units for different emission allowance levels. The proposed stochastic MILP approach proved both to be accurate and computationally acceptable, since the computation time scales up linearly with number of price scenarios, units and hours on the time horizon. This computational acceptance is due to the stochastic MILP being suitable to address parameter uncertainty via scenarios. As a final contribution of the approach to the decision making the cost of the spinning reserve is accessed, enabling a better trading on this reserve.

\section{References}

1. Li, Y.P., Huang, G.H.: Electric-Power Systems Planning and Greenhouse-Gas Emission Management Under Uncertainty. Energy Conv. Manag. 57, 173-182 (2012)

2. Wu, L., Shahidehpour, M., Li, T.: Stochastic Security-Constrained Unit Commitment. IEEE Trans. Power Syst. 22(2), 800-811 (2007)

3. Amjady, N., Nasiri-Rad, H.: Economic Dispatch Using an Efficient Real Coded Genetic Algorithm. IET Gener. Transm. Distrib. 3(3), 266-278 (2009)

4. Morales-España, G., Latorre, J.M., Ramos, A.: Tight and Compact MILP Formulation of Start-Up and Shut-Down Ramping in Unit Commitment. IEEE Trans. Power Syst. 28(2), 1288-1296 (2013) 
5. Yamin, H., Shahidehpour, M.: Self-Scheduling and Energy Competitive Electricity Markets. Electr. Power Syst. Res. 71(3), 203-209 (2004)

6. UCTE. Operation handbook: G3 Recommended Secondary Control Reserve (2004)

7. Collective Awareness Platforms for Sustainability and Social Innovation, http: / / ec. europa.eu/digital-agenda/collectiveawareness (accessed October 2013)

8. Senjyu, T., Shimabukuro, K., Uezato, K., Funabashi, T.: A Fast Technique for Unit Commitment Problem by Extended Priority List. IEEE Trans. Power Syst. 18, 882-888 (2003)

9. Dhillon, J.S., Kothari, D.P.: Economic-Emission Load Dispatch Using Binary Successive Approximation-Based Evolutionary Search. IET Gener. Trans. Distrib. 3(1), 1-16 (2009)

10. Chandrasekaran, K., Hemamalini, S., Simon, S.P., Padhy, N.: Thermal UC Using Binary/Real Coded Artificial Bee Colony Algorithm. Electr. Power Syst. Res. 84(1), 109-119 (2012)

11. Dieua, V.N., Ongsakul, W.: Augmented Lagrange Hopfield Network Based Lagrangian Relaxation for Unit Commitment. Int. J. Electr. Power Energy Syst. 33(3), 522-530 (2011)

12. Ouyang, Z., Shahidehpour, M.: A Hybrid Artificial Neural Network-Dynamic Programming Approach to Unit Commitment. IEEE Trans. Power Syst. 7(1), 236-242 (1992)

13. Kumar, V.S., Mohan, M.R.: Solution to Security Constrained Unit Commitment Problem Using Genetic Algorithm. Electr. Power Energy Syst. 32(2), 117-125 (2010)

14. Carrion, M., Arroyo, J.M.: A Computationally Efficient Mixed-Integer Linear Formulation for the Thermal Unit Commitment Problem. IEEE Trans. Power Syst. 21, 1371-1378 (2006)

15. Ostrowski, J., Anjos, M.F., Vannelli, A.: Tight Mixed-Integer Linear Programming Formulations for the Unit Commitment Problem. IEEE Trans. Power Syst. 27, 39-46 (2012)

16. Catalão, J.P.S., Pousinho, H.M.I., Mendes, V.M.F.: Short-Term Electricity Prices Forecasting in a Competitive Market by a Hybrid Intelligent Approach. Energy Conv. Manag. 52(2), 1061-1065 (2011)

17. Thermal Units Data, http://hmi-21.wix.com/thermalunits (accessed October 2013) 